\title{
Context-dependent Changes in Functional Connectivity of Auditory Cortices during the Perception of Object Words
}

\author{
Wessel O. van Dam, Eelco V. van Dongen, Harold Bekkering, \\ and Shirley-Ann Rueschemeyer
}

\begin{abstract}
Embodied theories hold that cognitive concepts are grounded in our sensorimotor systems. Specifically, a number of behavioral and neuroimaging studies have buttressed the idea that language concepts are represented in areas involved in perception and action [Pulvermueller, F. Brain mechanisms linking language and action. Nature Reviews Neuroscience, 6, 576-582, 2005; Barsalou, L. W. Perceptual symbol systems. Behavioral and Brain Sciences, 22, 577-660, 1999]. Proponents of a strong embodied account argue that activity in perception/ action areas is triggered automatically upon encountering a word and reflect static semantic representations. In contrast to what would be expected if lexical semantic representations are automatically triggered upon encountering a word, a number of studies failed to find motor-related activity for words with a putative action-semantic component [Raposo, A., Moss, H. E., Stamatakis, E. A., \& Tyler, L. K. Modulation of motor and premotor cortices by actions, action words and action sentences. Neuropsychologia, 47, 388-396, 2009; Rueschemeyer, S.-A., Brass, M., \& Friederici, A. D. Comprehending prehending: Neural correlates of processing verbs with motor stems. Journal of
\end{abstract}

\section{INTRODUCTION}

According to an embodied approach to cognition, language concepts are grounded in brain areas generally dedicated to perception and action. In other words, real-world experiences with word's referents form the basis for deriving lexical-semantic meaning (Barsalou, 1999, 2008; Pulvermueller, 1999, 2005; Glenberg, 1997). Different theoretical proposals have been put forth within the general embodied framework (Barsalou, 1999, 2008; Mahon \& Caramazza, 2008; Vigliocco, Vinson, Lewis, \& Garrett, 2004), which share the notion that sensorimotor brain regions play a role in language understanding but differ with regard to whether they ascribe a functional role to activation observed in modality-specific brain regions during language comprehension (for a review, see Meteyard, Cuadrado, Bahrami, \& Vigliocco, 2012). On one end of the spectrum are theories that argue that modalityspecific activations reflect postconceptual processes and

Radboud University Nijmegen
Cognitive Neuroscience, 19, 855-865, 2007]. In a recent fMRI study, Van Dam and colleagues [Van Dam, W. O., Van Dijk, M., Bekkering, H., \& Rueschemeyer, S.-A. Flexibility in embodied lexical-semantic representations. Human Brain Mapping, in press] showed that the degree to which a modality-specific region contributes to a representation considerably changes as a function of context. In the current study, we presented words for which both motor and visual properties (e.g., tennis ball, boxing glove) were important in constituting the concept. Our aim was to corroborate on earlier findings of flexible and context-dependent language representations by testing whether functional integration between auditory brain regions and perception/action areas is modulated by context. Functional connectivity was investigated by means of a psychophysiological interaction analysis, in which we found that bilateral superior temporal gyrus was more strongly connected with brain regions relevant for coding action information: (1) for Action Color words vs. Abstract words, and (2) for Action Color words presented in a context that emphasized action vs. a context that emphasized color properties.

are not a reflection of how meaning is represented (e.g., Mahon \& Caramazza, 2008). On the other end of the spectrum are theories that argue that the meaning of words is represented within sensorimotor brain areas (e.g., Pulvermueller \& Fadiga, 2010; Pulvermueller, 2005). Intermediate to conceptions at the two ends of the spectrum are theories that suggest that word meaning is mediated by initial connections to sensorimotor processes but which propose that other information is also important in forming a complete lexical-semantic representation (i.e., Barsalou, 2008; Simmons, Hamann, Harenski, Hu, \& Barsalou, 2008; Vigliocco et al., 2004).

Behavioral and neuroimaging results have substantiated the idea that areas typically involved in action and perception are also implicated in language processing (Hoenig et al., 2011; Kiefer, Sim, Herrnberger, Grothe, \& Hoenig, 2008; Kiefer, Sim, Liebich, Hauk, \& Tanaka, 2007). For example, it has been shown that executing an action interacts with comprehending sentences and single words denoting actions (Rueschemeyer, Pfeiffer, \& Bekkering, 2010; Van Dam, Rueschemeyer, Lindemann, 
\& Bekkering, 2010; Glenberg \& Kaschak, 2002). Likewise, Zwaan, Madden, Xaxley, and Aveyard (2004) have provided evidence that visual representations are activated during the comprehension of sentences describing a moving object. Findings from these behavioral experiments suggest that processes that play a role in action and perception are also involved in comprehending language about action (see Zwaan \& Kaschak, 2008, for a review). In a similar vein, neuroimaging studies have provided evidence that the neural motor system is recruited during comprehension of verbs that entail a motor component (Van Dam, Rueschemeyer, \& Bekkering, 2010; Hauk \& Pulvermueller, 2004) and words denoting manipulable objects (Saccuman et al., 2006; Chao \& Martin, 2000 ), whereas areas that have been linked to color processing are activated during the comprehension of words semantically related to color (Simmons et al., 2007; Pulvermueller \& Hauk, 2006; Kellenbach, Brett, \& Patterson, 2001; Martin, Haxby, Lalonde, Wiggs, \& Ungerleider, 1995). All the studies reviewed above provide evidence that perception and action have a common neural substrate with the processing of language semantically related to visual and motor information (for recent reviews on the role of modality-specific brain regions in language comprehension; see Borghi \& Cimatti, 2010; Andres, Olivier, \& Badets, 2008; Fisher \& Zwaan, 2008).

Although there is ample behavioral and neuroimag ing evidence for the selective involvement of modalityspecific brain regions during language processing, the automaticity and necessity of activation in perception and action areas in language processing remains a topic of debate. A number of neuroimaging studies have revealed that activation within modality-specific brain regions is elicited automatically, fast, and irrespective of the context. It has been demonstrated that category-specific activation occurs irrespective of attention to action words (Pulvermueller \& Shtyrov, 2006, 2009; Shtyrov, Hauk, \& Pulvermueller, 2004) can be observed as early as $\sim 200$ msec after word onset (Hoenig, Sim, Bochev, Herrnberger, \& Kiefer, 2008; Kiefer et al., 2008; Hauk \& Pulvermueller, 2004; Pulvermueller, Härle, \& Hummel, 2000) and also occurs for action verbs imbedded within idiomatic phrases (Boulenger, Hauk, \& Pulvermueller, 2009). These findings have been taken as evidence that words trigger activation in modality-specific brain regions in an automatic and bottom-up fashion.

According to a classical view of automaticity, automatic processes are triggered invariantly and independently of the current configuration of the cognitive system. Therefore, in this view, automaticity and context-dependent flexibility are in conflict with one another. It should be noted, though, that the finding that motor activations are called on in a flexible manner during word processing might not necessitate any contribution of intended, controlled processes. Kiefer and Martens (2010) proposed an attentional sensitization model of unconscious cognition, arguing that conscious and unconscious perception are guided by similar computational principles and susceptible to top-down modulation in a comparable manner (see also Martens, Ansorge, \& Kiefer, 2011). That is, the notion of automaticity and context-dependent flexibility are not conflicting but predicted by the attentional sensitization model.

In contrast to what would be expected if words would trigger activation in modality-specific brain regions in an automatic and bottom-up fashion, several studies have provided evidence for contextual influences on activation within modality-specific brain regions. Raposo, Moss, Stamatakis, and Tyler (2009) showed in an fMRI study a strong recruitment of motor/premotor cortices for the processing of action verbs in isolation (e.g., kick) or in literal sentences (e.g., kick the ball), whereas action verbs in an idiomatic context (e.g., kick the bucket) did not elicit such activations. In a similar vein, Rueschemeyer, Brass, and Friederici (2007) showed that processing of morphologically complex verbs built on motor stems (e.g., to comprehend) did not differentially engage the neural motor system as compared with morphologically complex verbs built on abstract stems (e.g., to consider). These findings challenge the idea that action words elicit activation in the motor system in an automatic and bottom-up fashion and suggest that the activation of meaning attributes of words depends on the context (i.e., sentence context in the case of Raposo et al., 2009, and morphological context in the case of Rueschemeyer et al., 2007). The abovementioned studies demonstrated a relative presence or absence of activation in motor and perceptual brain areas depending on the context, suggesting that embodied representations are flexible to some degree. These findings emphasize one of the greatest strengths of human language: Words can be used in a flexible manner, that is, depending on the context a word can have different meanings (in a similar vein, Bub \& Masson, 2010 showed context-dependent modulations of action priming effects). One way to realize such flexibility in our language system is by assuming that concepts are formed by both contextindependent and context-dependent properties (Barsalou, 1982). That is, context-independent properties form the core meaning of the concept and are activated by the word on all occasions, whereas context-dependent properties are only activated by relevant contexts in which the word appears and therefore allow for conceptual flexibility. In a recent study from our group, we investigated whether the neural response to comprehension of words denoting objects semantically related to both visual and motor information changed depending on the context in which these words were encountered. The results showed greater activation in brain regions relevant for coding action (motion) information (inferior parietal lobule [IPL], middle temporal gyrus [MTG]), providing that the context emphasized action properties and that the corresponding features were relevant aspects of the concept (Van Dam, Van Dijk, Bekkering, \& Rueschemeyer, in press; see also Hoenig et al., 2008). On a neural level, conceptual flexibility can 
be realized by assuming that concepts are built on multiple representational units, which can contribute to a concept varying as a function of the context in which a concept is accessed (Kiefer \& Pulvermueller, 2012). Such distributed semantic memory models are in sharp contrast with classical semantic memory models, which entail a localist representation format of concepts.

Neuroimaging studies on conceptual flexibility focused on the magnitude of brain activity; however, we might gain a better understanding of context effects in embodied lexical-semantic processing by investigating the interaction of auditory cortices and modality-specific brain regions during auditory word processing. One way to investigate the interaction of multiple brain regions in a particular task is by means of a psychophysiological interaction (PPI) analysis, which allows the delineation of interregional neural interactions by investigating how region-to-region coactivation patterns change as a function of psychological context (Friston, Frith, Liddle, \& Frackowiak, 1993). In the current study, we tried to corroborate on earlier findings of flexible and contextdependent language representations by testing whether patterns of functional connectivity between auditory cortices and perception/action areas are modulated by context. To this end, we conducted a PPI analysis using the bilateral auditory cortices (posterior portion of bilateral superior temporal gyrus [STG]) as our seed region. The goals of our study were twofold: First, we aimed to replicate previous results showing that functional connectivity between auditory brain regions and modalityspecific brain regions is stronger for concrete words (i.e., in our case action color words) than for abstract words (Ghio \& Tettamanti, 2010). Second, and most interestingly, we sought to determine whether the pattern of functional connectivity between auditory and modalityspecific brain regions is modified by encouraging participants to focus either on visual or motor properties of the denoted objects.

\section{METHODS}

\section{Participants}

Twenty students of Radboud University participated in the study, all of whom were right-handed and between 18 and 24 years of age ( $M=20.5, S D=2.2 ; 14$ women $)$. All participants had normal or corrected-to-normal vision and no history of neurological disorders. Beforehand, all participants were informed about the experimental procedures, were given practice trials, and signed informed consent. Afterward, all students were awarded $€ 12.5$ for participating. The data from one participant were excluded from analysis because of technical problems during the recording. The data of four additional participants were excluded, as they did not meet the criteria for the PPI analysis (see fMRI Data Analysis section below). The experiment was conducted in accordance with the national legislation for protection of human volunteers in research settings and the Helsinki Declaration of 1975, revised in 2004.

\section{Stimuli}

One hundred twenty (120) words were used as experimental stimuli (critical stimuli with English translations can be seen in Table 1). Twenty-five belonged to (1) the Action Color condition, that is, words denoting objects that are associated with both a specific action and color (e.g., boxing glove, tennis ball); 25 belonged to (2) the Abstract word condition, that is, words denoting abstract concepts (e.g., magic, justice); 20 constituted catch trials; and the remaining 50 stimuli were conditions of no interest for the current analysis. Details of those stimuli and analyses pertaining to them can be seen in Van Dam et al. (in press).

The validity and psycholinguistic properties of the experimental stimuli were tested using a prescanning questionnaire. Ten participants who did not take part in the subsequent scanning session were asked to rate critical stimuli on (1) actions associated with the word's referent, (2) colors associated with the word's referent, (3) familiarity (5-point scale: $1=$ unfamiliar, $5=$ very familiar), (4) comprehensibility ( $1=$ not comprebensible, $5=$ very comprebensible), (5) frequency ( $1=$ I use this word very rarely, $5=$ I use this word very often), and (6) imageability $(1=$ very difficult to image, $5=$ very easy to image $)$ Importantly, participants consistently indicated that Action Color words denoted objects used primarily with the hand/arm and were highly associated with one specific color. Furthermore, the results of the questionnaire showed that objects were highly similar across conditions with respect to familiarity with the word (Action/Color: $M=4.96$; Abstract: $M=4.98, t(9)=1.41, p>.150)$, comprehensibility (Action/Color: $M=4.97$; Abstract: $M=$ $4.95, t(9)=1.77, p>.100$ ), and frequency (Action/Color: $M=1.95$; Abstract: $M=2.31, t(9)=2.01, p>.05)$. Objects differed across conditions with respect to imageability (Action/Color: $M=4.94$; Abstract: $M=3.13, t(9)=6.28$, $p<.001)$. To obtain an objective measure of word frequency, we also calculated the mean frequency per million for each condition using the SUBTLEX database (Keuleers, Brysbaert, \& New, 2010). This value was 6.52 for the Action/ Color words and 28.13 for Abstract words. Independent sample $t$ tests indicated that there were no reliable differences in the mean frequency per million between the two experimental conditions, $t(48)=1.42, p>.150$. Thus, stimuli were matched for relevant linguistic parameters, familiarity, comprehensibility, and frequency. Words were also matched across conditions for length (Action Color: $M=7.9$ letters; Abstract: $M=9.0$ letters, $t(48)=1.46, p>$ .150 ), and for word duration (Action Color: $M=895 \mathrm{msec}$; Abstract: $M=872$ msec, $t(48)=1.54, p>.100)$. Participants confirmed that Action Color words denoted objects that are highly associated with an action (other than a foot action), whereas Abstract words were not associated with an action (percentage of yes responses: Action 
Table 1. Experimental Stimuli

\begin{tabular}{|c|c|c|c|c|c|}
\hline \multicolumn{2}{|c|}{ Action/Color } & \multicolumn{2}{|c|}{ Abstract Word } & \multicolumn{2}{|c|}{ Catch Items } \\
\hline Bakpapier & Waxed paper & Beperking & Restriction & Dollarbiljet & Dollar bill \\
\hline Bezem & Broom & Beroepsgroep & Professional group & Glasbak & Bottle bank \\
\hline Bokshandschoen & Boxing glove & Bijzonderheid & Peculiarity & Gras & Grass \\
\hline Cello & Cello & Breuk & Fraction & Krokodil & Crocodile \\
\hline Deegrol & Rolling pin & Commissie & Commission & Legerbroek & Army jeans \\
\hline Deurklink & Door latch & Creativiteit & Creativity & Legerhelm & Army belmet \\
\hline Dobbelsteen & Dice & Criminaliteit & Criminality & Legeruniform & Army uniform \\
\hline Drumstok & Drum stick & Crisis & Crisis & Pooltafel & Pool table \\
\hline Gitaar & Guitar & Detail & Detail & Regenlaars & Jackboot \\
\hline Lucifer & Match & Economie & Economy & Voetbalveld & Soccer field \\
\hline Mascara & Mascara & Gerechtigheid & Justice & Gaspedaal & Gas pedal \\
\hline Nagelknipper & Nail clipper & Gigabyte & Gigabyte & Skateboard & Skateboard \\
\hline Paperclip & Paperclip & Goedkoop & Cheap & Laars & Boot \\
\hline Pincet & Tweezers & Kwartaal & Quarter & Schaats & Skate \\
\hline Prittstift & Glue stick & Maatschappij & Society & Pianopedaal & Piano pedal \\
\hline Schroef & Screw & Magie & Magic & Ski & $S k i$ \\
\hline Sleutel & Key & Oorlog & War & Trampoline & Trampoline \\
\hline Spijker & Nail & Percentage & Percentage & Trapper & Pedal \\
\hline Tennisbal & Tennis ball & Probleem & Problem & Voetbal & Football \\
\hline Toiletpapier & Toilet paper & Spelregel & Rule of the game & Waterfiets & Pedal boat \\
\hline Viool & Violin & Verzekering & Insurance & & \\
\hline Wasbak & Sink & Welvaart & Prosperity & & \\
\hline Winkelwagen & Shopping cart & Werkgeheugen & Working memory & & \\
\hline Zaag & Saw & Winst & Profit & & \\
\hline Zwaard & Sword & Wraakzucht & Revengefulness & & \\
\hline
\end{tabular}

English translations are printed in italics.

Color: $M=84.8$; Abstract: $M=3.6$, all means significantly differed from $50 \%$ as indicated by one-sample $t$ tests; all ps < .001). In addition, participants confirmed that if a word was associated with an action, it was consistently rated as a hand/arm action (Action Color: $M=98.4$, means significantly differed from $50 \%$ as indicated by one-sample $t$ tests; all $p s<.001)$. Additionally, participants confirmed that Action Color words denoted objects that are highly associated with a color (other than green), whereas Abstract words were not associated with a color (percentage of yes responses: Action Color: $M=74.8$; Abstract: $M=2.4$, all means significantly differed from $50 \%$ as indicated by onesample $t$ tests; all $p s<.005)$. In addition, participants confirmed that if a word was associated with a color, it was consistently rated as associated with the same specific color across participants (Action Color: $M=91.0$, means significantly differ from $50 \%$ as indicated by one-sample $t$ tests; all $p s<.001)$.

All words and questions were presented auditorily by a female voice over MR-compatible headphones. After recording the spoken stimuli, they were segmented and equalized in amplitude using Cool Edit Pro 2.1 (Syntrillium Software Corporation).

\section{Procedure}

The order of stimulus presentation was randomized for each participant. All participants heard all experimental stimuli. A single trial lasted $8 \mathrm{sec}$ and constituted the presentation of a single word. Stimuli were presented in miniblocks of five items. In half of the blocks, participants had to answer a question that focused on the 
color attributes of the object ("Does this object have a green color?'), whereas in the other half of the blocks participants had to answer a question that focused on the action attributes of the object ("Is the object associated with an action performed with your feet?'). A variable jitter time of $0,500,1000$, or 1500 msec was included at the beginning of each trial to enhance the temporal resolution of the acquired signal. Following this, a fixation cross was presented in the center of the screen; the auditory stimulus word was presented $400 \mathrm{msec}$ after the onset of the fixation cross. The fixation cross was visible during word presentation and remained visible until 2000 msec after word offset. Hereafter, a variable intertrial interval filled the remaining time, so that every trial lasted exactly $8000 \mathrm{msec}$. The ISI was randomly jittered. Participants were instructed to listen to all words carefully and to perform a go/no-go semantic categorization task, in which go responses (by means of a button press) should be made only to words denoting objects that were associated with either a green color or a foot action (catch items). In this way, we ensured that participants semantically processed all words (i.e., to decide to answer or not, participants had to comprehend the words) while keeping critical stimuli free of motor execution artifacts. In 20 trials, a fixation cross was presented for the full $8 \mathrm{sec}$ "null trial". To ensure that our results are not driven by acoustic, phonological, or lexical factors, we presented each word twice. Presentation order of a specific word (i.e., in the color judgment or action judgment condition) was randomized both within and across participants.

\section{fMRI Data Acquisition}

Functional images were acquired on a Siemens TRIO 3.0 T MRI system (Siemens Healthcare, Erlangen, Germany) equipped with EPI capabilities, using a birdcage head coil for radio frequency transmission and signal reception. BOLD-sensitive functional images were acquired using a single-shot gradient EPI sequence (echo time/repetition time $=31 / 2000 \mathrm{msec} ; 31$ axial slices in ascending order, voxel size $=3.5 \times 3.5 \times 3.5)$. High-resolution anatomical images were acquired using an MPRAGE sequence (echo time $=3.03$, voxel size $=1 \times 1 \times 1 \mathrm{~mm}, 192$ sagittal slices, field of view $=256$ ).

\section{fMRI Data Analysis}

Functional data were preprocessed and analyzed with SPM8 (Statistical Parametric Mapping, www.fil.ion.ucl.ac.uk/spm). Preprocessing involved removing the first three volumes to allow for T1 equilibration effects. Rigid body registration along three translations and three rotations was applied to correct for small head movements. Subsequently, the time series for each voxel was realigned temporally to acquisition of the middle slice (Slice 16) to correct for slice timing acquisition delays. Images were normalized to a standard EPI template centered in Montreal Neurological Institute space and resampled at an isotropic voxel size of $2 \mathrm{~mm}$.
The normalized images were smoothed with an isotropic 8-mm FWHM Gaussian kernel. The ensuing preprocessed fMRI time series were analyzed on a subject-by-subject basis using an event-related approach in the context of the general linear model with regressors for each condition (Action Color $[\mathrm{AC}]$, Action $[\mathrm{A}]$, Color $[\mathrm{C}]$, and Abstract [Abs]) as well as the error trials, catch trials, and null trials (Null) convolved with a canonical hemodynamic response function. The parameters from the motion correction procedure were included in the model as effects of no interest.

The portion of the STG sensitive to auditory word processing was identified bilaterally in each participant by using the contrast between Action Color words (AC) and the fixation baseline (null trials). Voxels that showed significant activity (at $p<.005, k>65$ ) during the processing of Action Color words (as compared with the fixation baseline) in bilateral STG were extracted using MarsBar (Brett, Anton, Valabregue, \& Poline, 2002). Four of the participants were excluded from further analyses, given that the processing of Action Color words as compared with the fixation baseline did not show voxels in bilateral STG to be significantly activated. Subsequently, we extracted the time course from the fMRI time series within the defined seed region using its first eigenvector. A PPI analysis (Friston et al., 1997; Friston, 1994) was conducted to assess whether neural interactions of the bilateral STG with other brain areas, change depending on word concreteness and context. After the extraction of the ROI time course (from the bilateral STG; the physiological variable) and the psychological vector of interest (written as -1 or 1 for Action Color words and Abstract words, respectively), the psychophysiological interaction term was computed. We repeated this procedure for the other vector of interest (action context vs. color context). All variables were temporally convolved with the canonical HRF. A high pass filter was implemented using a high cutoff period of $192 \mathrm{sec}$ to remove low-frequency effects from the time series without removing the inherent low frequency effects of interest.

A separate model was run for each psycho-physiological contrast, representing (1) the main effect of reading Action

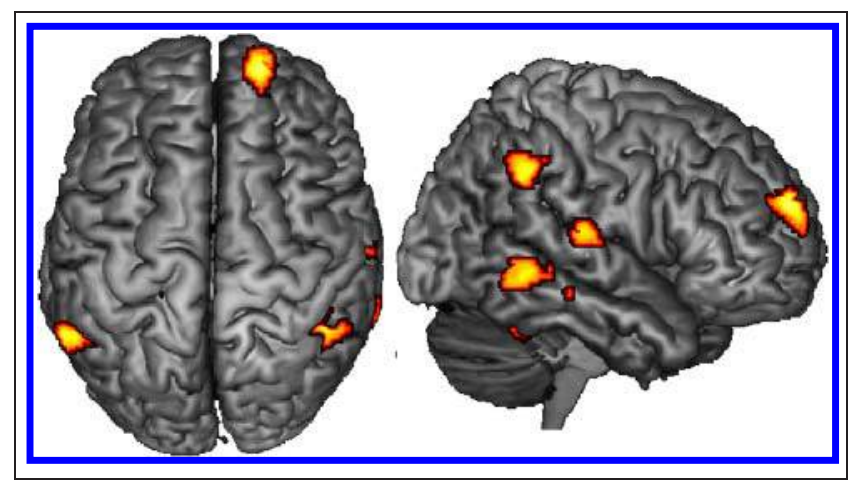

Figure 1. Increased functional connectivity of bilateral STG for Action Color words as compared with Abstract words (all voxels surviving the threshold of $p<.005, k>65$ ). 
Table 2. Brain Regions Showing Significantly More Functional Connectivity with the Bilateral STG for Action/Color, Action, Color than for Abstract Words $(p<.005, k>65)$

\begin{tabular}{|c|c|c|c|c|c|c|c|c|c|c|}
\hline \multirow[b]{2}{*}{ Brain Regions } & \multicolumn{5}{|c|}{ Action/Color $>$ Abstract } & \multicolumn{5}{|c|}{ Abstract $>$ Action/Color } \\
\hline & $Z \max$ & Extent (Voxels) & $x$ & $y$ & $z$ & $Z \max$ & Extent (Voxels) & $x$ & $y$ & $z$ \\
\hline R superior frontal gyrus & 3.55 & 149 & 18 & 62 & 26 & & & & & \\
\hline R caudate & 2.89 & 73 & 8 & 2 & 4 & & & & & \\
\hline $\mathrm{R}$ postcentral gyrus & 3.16 & 86 & 60 & -18 & 12 & & & & & \\
\hline $\mathrm{R}$ cingulate gyrus & 3.56 & 180 & 18 & -28 & 34 & & & & & \\
\hline L cingulate gyrus & 3.82 & 203 & -20 & -34 & 36 & & & & & \\
\hline R IPL & 3.65 & 219 & 46 & -44 & 38 & & & & & \\
\hline R MTG & 3.34 & 308 & 54 & -42 & -6 & & & & & \\
\hline L MTG & 3.46 & 252 & -56 & -52 & 4 & & & & & \\
\hline L supramarginal gyrus (IPL) & & & -45 & -50 & 36 & & & & & \\
\hline Cerebellum & 3.03 & 71 & 28 & -48 & -28 & & & & & \\
\hline
\end{tabular}

The maximum $Z$ scores, the cluster extent (in voxels), and the Montreal Neurological Institute coordinates are reported.

Color words (AC-Abs), (2) the effect of context on Action Color words (AC-abs, both for the Action and Color context), and (3) a direct contrast of Action Color words in the two contexts (AC in Action context-AC in Color context; $\mathrm{AC}$ in Color context-AC in Action context). Because individual functional data sets had been aligned to standard stereotactic reference space, a group analysis based on the contrast images could be performed. Single-participant contrast images were entered into a second-level random effects analysis for the critical contrast of interest. The group analysis consisted of a one-sample $t$ test across the contrast images of all participants, which indicated whether observed differences between conditions were significantly distinct from zero. To protect against false-positive activation, a double threshold was applied, by which only voxels with a $p<.005$, uncorrected, and volume exceeding 65 voxels were considered, resulting in a corrected $p$ value of $<.05$. The 65 -voxel threshold was determined by modeling the entire imaging volume, assuming an individual voxel Type I error and subsequently smoothing the volume with a Gaussian kernel. To achieve the desired correction for multiple comparisons, we calculated the probability associated with each cluster extent across 1000 Monte Carlo simulations (Slotnick, Moo, Segal, \& Hart, 2003; Forman et al., 1995).

\section{RESULTS}

\section{Seed Connectivity Analysis}

To test whether functional connectivity was observed between auditory regions and areas involved in lexicalsemantic processing during the perception of words that denoted objects associated with action and color information, we conducted a connectivity analysis using bilateral STG as seed region.

\section{Main Effect of Concreteness (Action Color vs. Abstract Words)}

We tested whether functional connectivity of auditory brain regions and brain areas involved in lexical-semantic

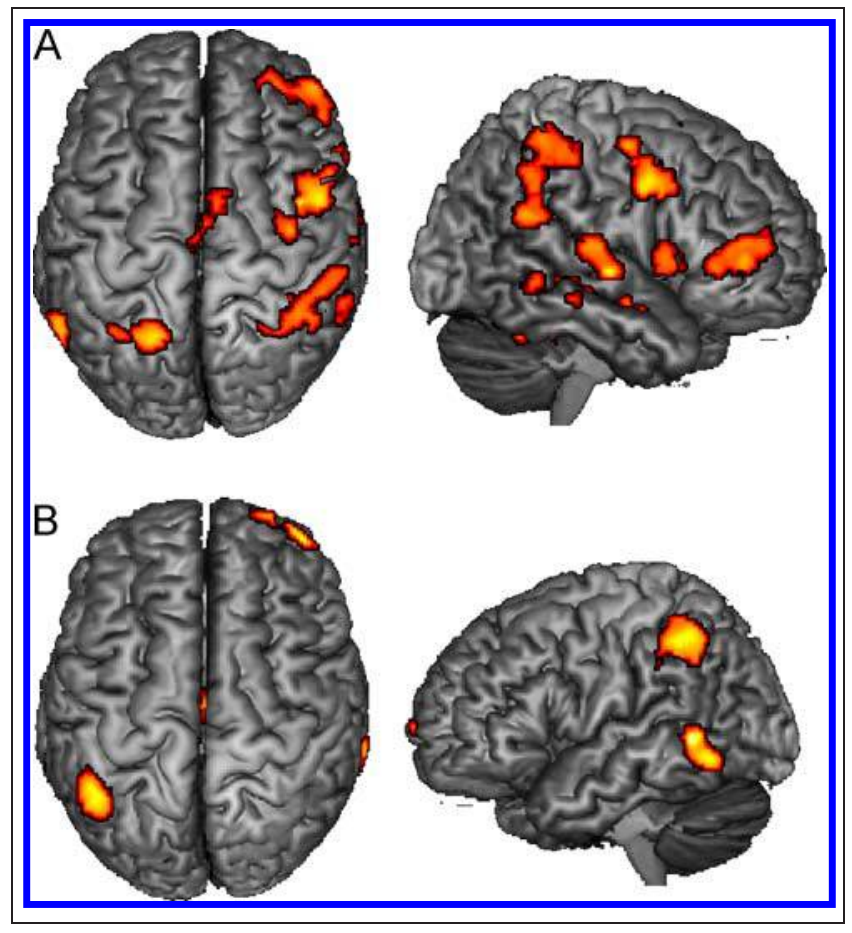

Figure 2. (A) Increased functional connectivity of bilateral STG for Action Color words in the action context as compared with Abstract words in the action context (all voxels surviving the threshold of $p<.005, k>65$ ). (B) Increased functional connectivity of bilateral STG for Action Color words in the color context as compared with Abstract words in the color context (all voxels surviving the threshold of $p<.005, k>65)$. 
Table 3. Brain Regions Showing Significantly More Functional Connectivity with the Bilateral STG for Action/Color than for Abstract Words for the Action and Color Context $(p<.005, k>65)$

\begin{tabular}{|c|c|c|c|c|c|c|c|c|c|c|}
\hline \multirow[b]{2}{*}{ Brain Regions } & \multicolumn{5}{|c|}{ Action Color (ac) > Abstract (ac) } & \multicolumn{5}{|c|}{ Action Color (co) > Abstract (co) } \\
\hline & $Z \max$ & Extent (Voxels) & $x$ & $y$ & $z$ & $Z \max$ & Extent (Voxels) & $x$ & $y$ & $z$ \\
\hline R MFG & & & & & & 3.80 & 82 & 42 & 58 & 8 \\
\hline \multirow[t]{2}{*}{ R IFG (spreading into precentral gyrus) } & 3.33 & 464 & 48 & 48 & 4 & & & & & \\
\hline & 4.38 & 487 & 38 & 4 & 34 & & & & & \\
\hline Caudate & 3.28 & 107 & 14 & 18 & 4 & & & & & \\
\hline Front inferior operculum & 3.05 & 121 & 56 & 14 & 4 & & & & & \\
\hline MFG & 3.01 & 81 & 8 & 6 & 56 & & & & & \\
\hline R STG & 3.94 & 191 & 64 & -10 & 0 & & & & & \\
\hline Thalamus & 3.69 & 156 & 12 & -20 & 12 & & & & & \\
\hline Midbrain & 3.09 & 87 & 4 & -28 & -22 & & & & & \\
\hline L cingulate gyrus & 3.75 & 325 & -16 & -34 & 36 & 3.77 & 155 & -2 & -14 & 28 \\
\hline $\mathrm{R}$ cingulate gyrus & 3.59 & 291 & 18 & -26 & 40 & & & & & \\
\hline L STG (spreading into IPL) & 3.49 & 149 & -60 & -54 & 18 & & & & & \\
\hline L IPL & & & & & & 3.53 & 429 & -46 & -44 & 46 \\
\hline \multirow[t]{2}{*}{ R IPL } & 3.32 & 108 & 58 & -46 & 24 & & & & & \\
\hline & 3.31 & 564 & 34 & -48 & 52 & & & & & \\
\hline L MTG & & & & & & 4.03 & 254 & -54 & -48 & 0 \\
\hline R MTG & & & & & & 3.80 & 82 & 64 & -48 & -8 \\
\hline Postcentral gyrus & 3.67 & 173 & -20 & -50 & 72 & & & & & \\
\hline \multirow[t]{2}{*}{ Cerebellum } & 3.63 & 281 & 22 & -54 & -24 & & & & & \\
\hline & 3.61 & 189 & -30 & -76 & -30 & & & & & \\
\hline $\mathrm{L}$ precuneus & & & & & & 3.01 & 78 & -8 & -68 & 38 \\
\hline $\mathrm{R}$ precuneus & 3.34 & 91 & 16 & -70 & 22 & & & & & \\
\hline
\end{tabular}

The maximum $Z$ scores, the cluster extent (in voxels), and the Montreal Neurological Institute coordinates are reported.

processing was modulated by word concreteness. Bilateral STG connectivity was substantially greater for Action Color words (e.g., boxing glove) than for Abstract words (e.g., magic) with the right superior frontal gyrus, right caudate, right postcentral gyrus, bilateral cingulate gyri, right IPL, right MTG, left MTG (spreading into the supramarginal gyrus), and the cerebellum (Figure 1; Table 2). Contrasting functional connectivity of Abstract with Action Color words did not yield any significant results.

Subsequently, we tested for the modulating effect of word concreteness on functional connectivity of auditory brain regions and brain areas involved in lexical-semantic processing. We did so separately for the action and the color context. Within the action context we observed that bilateral STG connectivity was substantially greater for Action Color words than for Abstract words with the right inferior frontal gyrus (IFG; spreading into precentral gyrus),

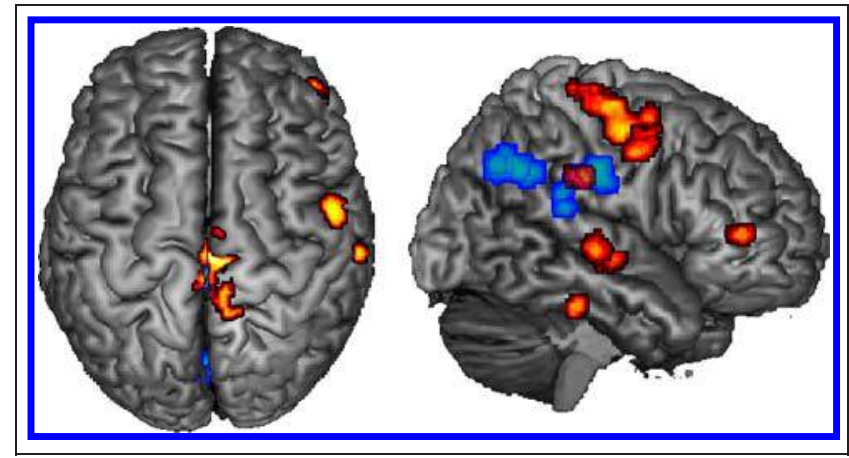

Figure 3. Increased functional connectivity of bilateral STG for Action Color words in the action context as compared with Action Color words in the color context (in red); increased functional connectivity of bilateral STG for Action Color words in the color context as compared with Action Color words in the action context (in blue; all voxels surviving the threshold of $p<.005, k>65$ ). 
caudate, frontal inferior operculum, right medial frontal gyrus (MFG), right STG, thalamus, midbrain, bilateral cingulate gyri, left STG (spreading into IPL), right IPL, left postcentral gyrus, cerebellum, and right precuneus. Within the color context, we observed that bilateral STG connectivity was substantially greater for Action Color words than for Abstract words with the right MFG, left cingulate gyrus, left IPL, bilateral MTG, and the left precuneus (Figure 2; Table 3).

\section{Main Effect of Context}

We tested whether functional connectivity of auditory brain regions and brain areas involved in lexical-semantic

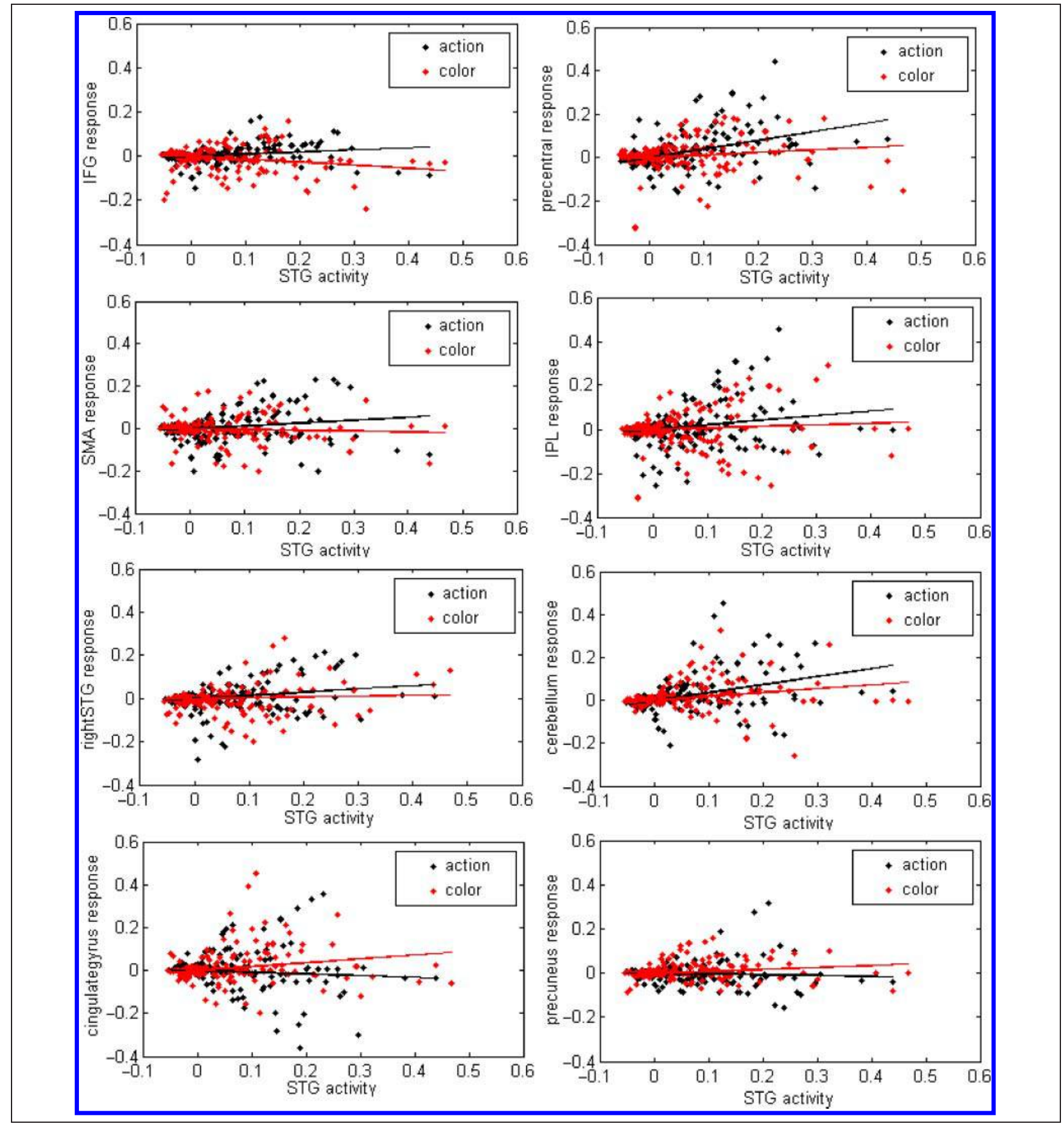

Figure 4. Results of the PPI analyses from a representative subject (number 12). Measurements during the presentation of action color (AC) words in an action context are indicated by black diamonds. Measurements during the presentation of AC words in a color context are indicated by red diamonds. The regression slopes of the respective conditions are indicated by black and red lines. The response (in arbitrary units) in our target regions (see Figure 3 and Table 4 ) is displayed as a function of the response in the bilateral STG (the seed region). 
Table 4. Brain Regions Showing Significantly More Functional Connectivity with the Bilateral STG for Action/Color Words in the Action Context versus the Color Context and for Action/Color Words in the Color Context versus the Action Context $(p<.005, k>65)$

\begin{tabular}{|c|c|c|c|c|c|c|c|c|c|c|}
\hline \multirow[b]{2}{*}{ Brain Regions } & \multicolumn{5}{|c|}{ Action Color (ac) > Action Color (co) } & \multicolumn{5}{|c|}{ Action Color (co) > Action Color (ac) } \\
\hline & $Z \max$ & Extent (Voxels) & $x$ & $y$ & $z$ & $Z \max$ & Extent (Voxels) & $x$ & $y$ & $z$ \\
\hline R IFG & 3.18 & 81 & 44 & 46 & 4 & & & & & \\
\hline $\mathrm{R}$ precentral gyrus & 3.15 & 103 & 54 & 2 & 38 & & & & & \\
\hline R SMA & 3.68 & 383 & 4 & -16 & 54 & & & & & \\
\hline R IPL & 3.00 & 73 & 60 & -20 & 26 & & & & & \\
\hline R STG & 3.43 & 125 & 52 & -20 & -2 & & & & & \\
\hline $\mathrm{L}$ cingulate gyrus & & & & & & 3.75 & 311 & -6 & -26 & 34 \\
\hline $\mathrm{L}$ precuneus & & & & & & 3.39 & 347 & -18 & -58 & 36 \\
\hline Cerebellum & 3.15 & 65 & -10 & -36 & -20 & & & & & \\
\hline
\end{tabular}

The maximum $Z$ scores, the cluster extent (in voxels), and the Montreal Neurological Institute coordinates are reported.

processing was modulated by the context in which Action Color words were presented (i.e., action versus color context). Bilateral STG connectivity was substantially greater for Action Color words in the action context than for Action Color words in the color context with the right IFG, right precentral gyrus, right SMA, right IPL, right STG, and the cerebellum. In contrast, bilateral STG connectivity was substantially greater for Action Color words in the color context than for Action Color words in the action context with the left cingulate gyrus and the left precuneus (Figures 3 and 4; Table 4).

\section{Main Effect of Context: Using Left Fusiform Gyrus as Seed Region}

Lastly, we tested whether functional connectivity of the left fusiform gyrus (FFG) was modulated by the context in which Action Color words were presented (i.e., action versus color context). The left FFG was defined using the contrast of Action Color words as compared with Abstract words. Voxels that showed significant activity during the auditory presentation of Action Color as compared with Abstract words in the left FFG (within a sphere of $10 \mathrm{~mm}$ centered at $[-24-30-28]$ ) were extracted using Marsbar (Brett et al., 2002). Coordinates were taken from a previous study from our group (Van Dam et al., in press). Left FFG connectivity was substantially greater for Action Color words in the color context than for Action Color words in the action context in the corpus callosum, the left cingulate gyrus, the posterior portion of the right STG, and the right precuneus (Figure 5; Table 5).

\section{DISCUSSION}

The current experiment was designed to explore whether patterns of functional connectivity of the bilateral auditory cortices are invariant in response to a given word or if they change depending on the context in which a word is perceived. There are two main results of this study. First, words denoting objects associated with both actions and colors as compared with abstract words showed a greater functional connectivity of bilateral STG with right superior frontal gyrus, right caudate, right postcentral gyrus, right IPL, bilateral cingulate gyri, bilateral MTG, and the cerebellum. Second, Action Color words in an action context as compared with Action Color words in a color context showed a greater functional connectivity of bilateral STG with right IFG, right precentral gyrus, right SMA, right IPL, right STG, and the cerebellum. In contrast, Action Color words in a color context as compared with Action Color words in an action context showed greater functional connectivity of bilateral STG with left cingulate gyrus and left precuneus.

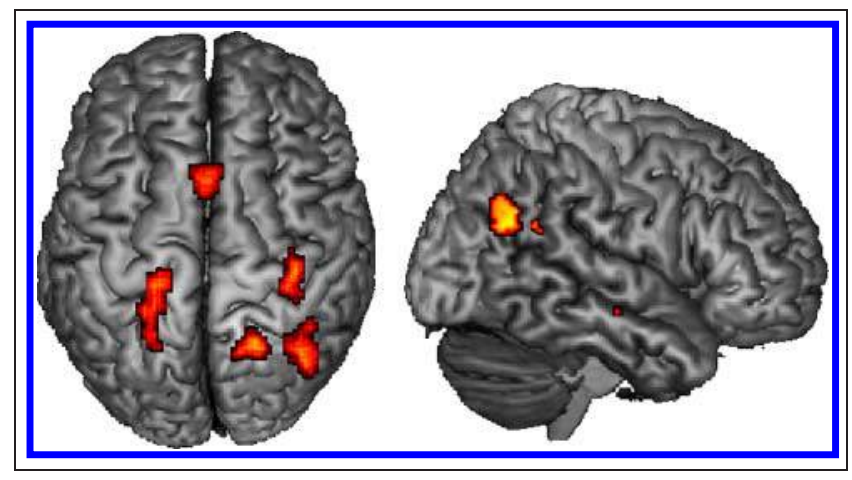

Figure 5. Increased functional connectivity of the left FFG for Action Color words in the color context as compared with Action Color words in the action context (all voxels surviving the threshold of $p<.005$, $k>65)$. 
Table 5. Brain Regions Showing Significantly More Functional Connectivity with the Left FFG for Action/Color Words in the Action Context versus the Color Context and for Action/Color Words in the Color Context versus the Action Context $(p<.005, k>65)$

\begin{tabular}{|c|c|c|c|c|c|c|c|c|c|c|}
\hline \multirow[b]{2}{*}{ Brain Regions } & \multicolumn{5}{|c|}{ Action Color (ac) > Action Color (co) } & \multicolumn{5}{|c|}{ Action Color (co) $>$ Action Color (ac) } \\
\hline & $Z \max$ & Extent (Voxels) & $x$ & $y$ & $z$ & $Z \max$ & Extent (Voxels) & $x$ & $y$ & $z$ \\
\hline Corpus Callosum & & & & & & 3.51 & 72 & 0 & 12 & 16 \\
\hline \multirow[t]{2}{*}{ Spreading into left cingulate gyrus } & & & & & & 3.73 & 180 & -20 & -30 & 28 \\
\hline & & & & & & 3.78 & 121 & 38 & -32 & -6 \\
\hline \multirow[t]{2}{*}{ R posterior STG } & & & & & & 3.61 & 170 & 40 & -58 & 26 \\
\hline & & & & & & & & 44 & -50 & 20 \\
\hline $\mathrm{R}$ precuneus & & & & & & 3.99 & 116 & 22 & -58 & 32 \\
\hline
\end{tabular}

The maximum $Z$ scores, the cluster extent (in voxels), and the Montreal Neurological Institute coordinates are reported.

\section{Concreteness}

The results of our study demonstrate that for words with both a putative action and color feature, bilateral STG shows stronger connectivity to brain regions relevant for coding action information (postcentral gyrus, IPL, bilateral MTG) than for abstract words. These findings are consistent with a study by Ghio and Tettamanti (2010), showing that connectivity between auditory and motor regions was increased during listening to action-related sentences, as compared with listening to abstract sentences. These findings are also in line with several neuroimaging studies that focused on differences in the magnitude of brain activity elicited by concrete versus abstract concepts (Van Dam et al., in press; Desai, Binder, Conant, \& Seidenberg, 2010; Van Dam, Rueschemeyer, \& Bekkering, 2010; Rueschemeyer et al., 2007). Contrasting functional connectivity of Abstract with Action Color words did not yield any significant results.

\section{Context-dependent Connectivity}

Our results demonstrate that for words with both a putative action and color feature, bilateral STG connectivity changed depending on the context. Specifically, if participants were encouraged to think about action as compared with color properties of the denoted object, greater bilateral STG connectivity was observed with brain regions relevant for coding action information (precentral gyrus, SMA, IPL). Conforming with embodied theories of cognition, several studies have shown that action-related language elicits activation within precentral gyrus (Pulvermueller, Hauk, Nikulin, \& Ilmoniemi, 2005; de Lafuente \& Romo, 2004; Hauk, Johnsrude, \& Pulvermueller, 2004), SMA (Desai et al., 2010), and IPL (Van Dam et al., in press; Desai et al., 2010; Van Dam, Rueschemeyer, \& Bekkering, 2010; Tettamanti et al., 2005). The precentral gyrus (motor cortex) has a pivotal role in the execution of movements (Graziano, Taylor, \& Moore, 2002) and is somatotopically organized (Penfield \& Rasmussen, 1950). The SMA has an important role in the selection, planning, and execution of movements (Lau, Rogers, Haggard, \& Passingham, 2004; Picard \& Strick, 1996). The IPL has been shown to code precise movement kinematics during movement execution (Iacoboni et al., 1999, 2001). In addition, it has been demonstrated that IPL neurons are sensitive to the final goal of a grasping action (Fogassi et al., 2005). That is, the IPL is critical for the representation of action plans and goals (see also Hamilton \& Grafton, 2006).

In a previous study from our group (Van Dam et al., in press), we observed that the left FFG, a brain region that has been linked to color processing (Simmons et al., 2007; Kellenbach et al., 2001; Martin et al., 1995), was activated for Action Color words. However, in contrast to what would be expected, we did not observe greater bilateral STG connectivity with the left FFG if participants were encouraged to think about color as compared with action properties of the denoted object. Action Color words in a color context as compared with Action Color words in an action context showed greater functional connectivity of bilateral STG with left cingulate gyrus and left precuneus. That is, no difference was observed between language contexts in connectivity of bilateral STG and the left FFG. This finding might reflect a crucial difference between action and color information. That is, action features are variant, with different types of actions becoming relevant for the same tool in different sentence (and action) contexts, whereas color is a static visual property. To explore whether we are able to find a contextual modulation of the functional coupling of a brain region that has shown to be relevant in processing color information with other brain regions, we conducted a post hoc analysis using the left FFG as our seed region. Left FFG connectivity was substantially greater for Action Color words in the color context than for the same words in the action context in the Corpus Callosum, the left cingulate gyrus, the posterior portion of the right STG, and the right precuneus. Contrasting FFG functional connectivity of the action with the color context did not yield any significant results.

In the current study, we found greater functional connectivity of bilateral STG to precentral gyrus, SMA, and 
IPL in response to Action Color words presented in a context that emphasized action properties as compared with the same words presented in a context that emphasized color properties. In addition, we found greater functional connectivity of left FFG to the posterior portion of the right STG in response to Action Color words presented in a context that emphasized color properties as compared with the same words presented in a context that emphasized action properties. These findings provide compelling evidence that the functional coupling of brain regions involved in auditory word processing (Burton, Noll, \& Small, 2001) with brain areas coding action/color-related properties is modulated by the relevance of modalityspecific properties in a given context. This finding is consistent with and corroborates on findings from previous studies that showed that the magnitude of brain activity within modality-specific brain regions in response to a specific word considerably changes as a function of context (Van Dam et al., in press; Hoenig et al., 2008).

\section{Acknowledgments}

The authors thank Paul Gaalman and Pascal de Water for technical support. The authors also thank Alex Brandemeyer, Sybrine Bultena, and Rutger Vlek for their help in making the recordings. The study was supported by a NWO-Veni grant (No. 275-89-002) to S.-A. R. and an NWO-Vici grant (No. 453-05-001) to H. B.

Reprint requests should be sent to Wessel O. van Dam, Donders Centre for Brain, Cognition and Behaviour, Centre for Cognition, Montessorilaan 3, $6525 \mathrm{HR}$, Nijmegen, The Netherlands, or via e-mail:w.vandam@donders.ru.nl.

\section{REFERENCES}

Andres, M., Olivier, E., \& Badets, A. (2008). Actions, words, and numbers. Current Directions in Psychological Science, 17, 313-317.

Barsalou, L. W. (1982). Context-independent and contextdependent information in concepts. Memorv and Cognition. 10, 82-93.

Barsalou, L. W. (1999). Perceptual symbol systems. Behavioral and Brain Sciences, 22, 577-660.

Barsalou, L. W. (2008). Grounded cognition. Annual Review of Psvchology, 59, 617-645.

Borghi, A. M., \& Cimatti, F. (2010). Embodied cognition and beyond: Acting and sensing the body. Neuropsychologia. 48, 763-773.

Boulenger, V., Hauk, O., \& Pulvermueller, F. (2009). Grasping ideas with your motor system: Semantic somatotopy in idiom comprehension. Cerebral Cortex, 19, 1905-1914.

Brett, M., Anton, J., Valabregue, R., \& Poline, J. (2002). Region of interest analysis using SPM toolbox. Neuroimage, 16, 1.

Bub, D. N., \& Masson, M. E. J. (2010). Grasping beer mugs: On the dynamics of alignment effects induced by handled objects. Journal of Experimental Psychologv: Human Perception and Performance, 36, 341-358.

Burton, M. W., Noll, D. C., \& Small, S. L. (2001). The anatomy of auditory word processing: Individual variability. Brain and Lanquage, 77, 119-131.

Chao, L. L., \& Martin, A. (2000). Representation of manipulable manmade objects in the dorsal stream. Neuroimage, 12 , 478-484. de Lafuente, V., \& Romo, R. (2004). Language abilities of motor cortex. Neuron, 41, 178-180.

Desai, R. H., Binder, J. R., Conant, L. L., \& Seidenberg, M. S. (2010). Activation of sensory-motor areas in language comprehension. Cerebral Cortex, 20, 468-478.

Fisher, M., \& Zwaan, R. (2008). Embodied language: A review of the role of the motor system in language comprehension. The Ouarterly Iournal of Experimental Psvchologv, 61, 825-850.

Fogassi, L., Ferrari, P. F., Gesierich, B., Rozzi, S., Chersi, F., \& Rizzolatti, G. (2005). Parietal lobe: From action organization to intention understanding. Science, 308, 662-667.

Forman, S., Cohen, J., Fitzgerald, M., Eddy, W., Mintun, M., \& Noll, D. (1995). Improved assessment of significant activation in functional magnetic resonance imaging (fMRI): Use of a cluster-size threshold. Masnetic Resonance in Medicine, 33, 637-647.

Friston, K. (1994). Functional and effective connectivity in neuroimaging: A synthesis. Human Brain Mapping 1, 56-78.

Friston, K. J., Buechel, C., Fink, G. R., Morris, J., Rolls, E., \& Dolan, R. (1997). Psychophysiological and modulatory interactions in neuroimaging. Neuroimage, 6, 218-229.

Friston, K. J., Frith, C. D., Liddle, P. F., \& Frackowiak, R. S. (1993). Functional connectivity: The principal component analysis of large (PET) data sets. Journal of Cerebral Blood Flow and Metabolism, 13, 5-14.

Ghio, M., \& Tettamanti, M. (2010). Semantic domain-specific functional integration for action-related vs. abstract concepts. Brain and Lanquage, 112, 223-232.

Glenberg, A. M. (1997). What memory is for. Behavioral and Brain Sciences, 20, 1-55.

Glenberg, A., \& Kaschak, M. (2002). Grounding language in action. Psychonomic Bulletin and Review, 9, 558-565.

Graziano, M. S., Taylor, C. S., \& Moore, T. (2002). Complex movements evoked by microstimulation of precentral cortex. Neuron, 34, 841-851.

Hamilton, A. F. C., \& Grafton, S. T. (2006). Goal representation in human anterior intraparietal sulcus. The Journal of Cognitive Neuroscience, 26, 1133-1137.

Hauk, O., Johnsrude, I., \& Pulvermueller, F. (2004). Somatotopic representation of action words in human motor and premotor cortex. Neuron, 41, 301-307.

Hauk, O., \& Pulvermueller, F. (2004). Neurophysiological distinction of action verbs in the fronto-central cortex. Human Brain Matping, 21, 191-201.

Hoenig, K., Müller, C., Herrnberger, B., Spitzer, M., Ehret, G., \& Kiefer, M. (2011). Neuroplasticity of semantic maps for musical instruments in professional musicians. Neuroimage, 56, 1714-1725.

Hoenig, K., Sim, E. J., Bochev, V., Herrnberger, B., \& Kiefer, M. (2008). Conceptual flexibility in the human brain: Dynamic recruitment of semantic maps from visual, motor, and motion-related areas. Journal of Cognitive Neuroscience, 20, 1799-1814.

Iacoboni, M., Koski, L. M., Brass, M., Bekkering, H., Woods, R. P., Dubeau, M. C., et al. (2001). Reafferent copies of imitated actions in the right superior temporal cortex. Proceedings of the National Academv of Sciences. U.S.A. 98, 13995-13999.

Iacoboni, M., Woods, R. P., Brass, M., Bekkering, H., Mazziotta, J. C., \& Rizzolatti, G. (1999). Cortical mechanisms of human imitation. Science, 286, 2526-2528.

Kellenbach, M. L., Brett, M., \& Patterson, K. (2001). Large, colorful or noisy? Attribute and modality-specific activations during retrieval of perceptual attribute knowledge. Cognitive. Affective \& Behavioral Neuroscience, 1, 207-221.

Keuleers, E., Brysbaert, M., \& New, B. (2010). SUBTLEX-NL: A new frequency measure for Dutch words based on film subtitles. Behavior Research Methods, 42, 643-650. 
Kiefer, M., \& Martens, U. (2010). Attentional sensitization of unconscious cognition: Task sets modulate subsequent masked semantic priming. Journal of Experimental Psvchologv: General, 139, 464-489.

Kiefer, M., \& Pulvermueller, F. (2012). Conceptual representations in mind and brain: Theoretical developments, current evidence and future directions. Cortex, 48, 805-825.

Kiefer, M., Sim, E.-J., Herrnberger, B., Grothe, J., \& Hoenig, M. (2008). The sound of concepts: Four markers for a link between auditory and conceptual brain systems. The Journal of Neuroscience, 28, 12224-12230.

Kiefer, M., Sim, E.-J., Liebich, S., Hauk, O., \& Tanaka, J. W. (2007). Experience-dependent plasticity of conceptual representations in human sensory-motor areas. Journal of Cognitive Neuroscience, 19, 525-542.

Lau, H. C., Rogers, R. D., Haggard, P., \& Passingham, R. E. (2004). Attention to intention. Science, 303, 1208-1210.

Mahon, B. Z., \& Caramazza, A. (2008). A critical look at the embodied cognition hypothesis and a new proposal for grounding conceptual content. Journal of Physiology Paris, 102, 59-70.

Martens, U., Ansorge, U., \& Kiefer, M. (2011). Controlling the unconscious: Attentional task sets modulate subliminal semantic and visuo-motor processes differentially. Psvchological Science, 22, 282-291.

Martin, A., Haxby, J. V., Lalonde, F. M., Wiggs, C. L., \& Ungerleider, L. G. (1995). Discrete cortical regions associated with knowledge of color and knowledge of action. Science, 210, 102-105.

Meteyard, L., Cuadrado, S. R., Bahrami, B., \& Vigliocco, G. (2012). Coming of age: A review of embodiment and neuroscience of semantics. Cortex, 48, 788-804.

Penfield, W., \& Rasmussen, T. (1950). The cerebral cortex of man. New York: Macmillan.

Picard, N., \& Strick, P. L. (1996). Medial wall motor areas: A review of their location and functional activation. Cerebral Cortex, 6, 342-353.

Pulvermueller, F. (1999). Words in the brain's language. Behavioral and Brain Sciences, 22, 253-336.

Pulvermueller, F. (2005). Brain mechanisms linking language and action. Nature Reviews Neuroscience, 6, 576-582.

Pulvermueller, F., \& Fadiga, L. (2010). Active perception: Sensorimotor circuits as a cortical basis for language understanding. Nature Reviews Neuroscience, 11, 351-360.

Pulvermueller, F., Härle, M., \& Hummel, F. (2000). Neurophysiological distinction of verb categories. NeuroReport, 11, 2789-2793.

Pulvermueller, F., \& Hauk, O. (2006). Category-specific conceptual processing of color and form in left frontotemporal cortex. Cerebral Cortex, 16, 1193-1201.

Pulvermueller, F., Hauk, O., Nikulin, V. V., \& Ilmoniemi, R. J. (2005). Functional links between motor and language systems. European Iournal of Neuroscience, 21, 793-797.

Pulvermueller, F., \& Shtyrov, Y. (2006). Language outside the focus of attention: The mismatch negativity as a tool for studying higher cognitive processes. Progress in Neurobiology, 79, 49-71.
Pulvermueller, F., \& Shtyrov, Y. (2009). Spatio-temporal signatures of large-scale synfire chains of speech: MEG evidence. Cerebral Cortex, 19, 79-88.

Raposo, A., Moss, H. E., Stamatakis, E. A., \& Tyler, L. K. (2009). Modulation of motor and premotor cortices by actions, action words and action sentences. Neuropsvchologia, 47, 388-396.

Rueschemeyer, S.-A., Brass, M., \& Friederici, A. D. (2007). Comprehending prehending: Neural correlates of processing verbs with motor stems. Iournal of Cognitive Neuroscience, 19, 855-865.

Rueschemeyer, S.-A., Pfeiffer, C., \& Bekkering, H. (2010). Body schematics: On the role of the body schema in embodied lexical-semantic representations. Neuropsychologia, 48, 774-781.

Saccuman, M. C., Cappa, S. F., Bates, E. A., Arevalo, A., Della Rosa, P., Danna, M., et al. (2006). The impact of semantic reference on word class: An fMRI study of action and object naming. Neuroimage, 32, 1865-1878.

Shtyrov, Y., Hauk, O., \& Pulvermueller, F. (2004). Distributed neuronal networks for encoding category-specific semantic information: The mismatch negativity to action words. European Iournal of Neuroscience, 19, 1083-1092.

Simmons, W. K., Hamann, S. B., Harenski, C. L., Hu, X. P., \& Barsalou, L. W. (2008). fMRI evidence for word association and situated simulation in conceptual processing. Journal of Pbusiology Paris, 102, 106-119.

Simmons, W. K., Ramjee, V., Beauchamp, M. S., McRae, K., Martin, A., \& Barsalou, L. W. (2007). A common neural substrate for perceiving and knowing about color. Neuropsychologia, 45, 2802-2810.

Slotnick, S. D., Moo, L. R., Segal, J. B., \& Hart, J. (2003). Distinct prefrontal cortex activity associated with item memory for visual shapes. Cognitive Brain Research, 17, 75-82.

Tettamanti, M., Buccino, G., Saccuman, M. C., Gallese, V., Danna, M., Scifo, P., et al. (2005). Listening to action-related sentences activates fronto-parietal motor circuits. Journal of Cognitive Neuroscience, 17, 273-281.

Van Dam, W. O., Rueschemeyer, S.-A., \& Bekkering, H. (2010). How specifically are action verbs presented in the neural motor system: An fMRI study. Neuroimage, 53, 1318-1325.

Van Dam, W. O., Rueschemeyer, S.-A., Lindemann, O., \& Bekkering, H. (2010). Context effects in embodied lexicalsemantic processing. Frontiers in Psychology, 1, 150.

Van Dam, W. O., Van Dijk, M., Bekkering, H., \& Rueschemeyer, S.-A. (in press). Flexibility in embodied lexical-semantic representations. Human Brain Mapping, doi: $10.1002 / \mathrm{hbm} .21365$.

Vigliocco, G., Vinson, D. P., Lewis, W., \& Garrett, M. (2004). Representing the meanings of object and action words: The featural and unitary semantic space hypothesis. Cognitive Psychology, 48, 422-488.

Zwaan, R. A., \& Kaschak, M. P. (2008). Language in the brain, body, and world. In M. Robbins \& M. Aydede (Eds.), Cambridge handbook of situated cognition (pp. 368-381). Cambridge: Cambridge University Press.

Zwaan, R. A., Madden, C. J., Xaxley, R. H., \& Aveyard, M. E. (2004). Moving words: Dynamic mental representations in language comprehension. Cognitive Science, 28, 611-619. 


\section{This article has been cited by:}

1. Barbara Tomasino, Franco Fabbro, Paolo Brambilla. 2014. How do conceptual representations interact with processing demands: An fMRI study on action- and abstract-related words. Brain Research 1591, 38-52. [CrossRef]

2. Michiel van Elk, Hein van Schie, Harold Bekkering. 2014. Action semantics: A unifying conceptual framework for the selective use of multimodal and modality-specific object knowledge. Physics of Life Reviews 11, 220-250. [CrossRef]

3. Barbara Tomasino, Dario Marin, Roberto Eleopra, Sara Rinaldo, Lettieri Cristian, Mucchiut Marco, Belgrado Enrico, Monica Zanier, Riccardo Budai, Massimo Mondani, Stanislao D’Auria, Miran Skrap, Franco Fabbro. 2014. To move or not to move: Subthalamic deep brain stimulation effects on implicit motor simulation. Brain Research . [CrossRef]

4. Jie Yang. 2014. Influences of motor contexts on the semantic processing of action-related language. Cognitive, Affective, \& Behavioral Neuroscience . [CrossRef]

5. Alessandro Innocenti, Elisa Stefani, Mariateresa Sestito, Maurizio Gentilucci. 2014. Understanding of action-related and abstract verbs in comparison: a behavioral and TMS study. Cognitive Processing 15, 85-92. [CrossRef]

6. Barbara Tomasino, Marta Maieron, Elisa Guatto, Franco Fabbro, Raffaella Ida Rumiati. 2013. How are the motor system activity and functional connectivity between the cognitive and sensorimotor systems modulated by athletic expertise?. Brain Research 1540, 21-41. [CrossRef]

7. Jie YangContext Effects on Embodied Representation of Language Concepts 1-22. [CrossRef] 